\title{
Access and metro network convergence for flexible end to end network design [invited]
}

\author{
M. Ruffini, M. Achouche, A. Arbelaez, R. Bonk, A. Di Giglio, N. J. Doran, M. Furdek, R. Jensen, J. Montalvo, \\ N. Parsons, T. Pfeiffer, L. Quesada, C. Raack, H. Rohde, M. Schiano, G. Talli, P. Townsend, R. Wessaly, L. \\ Wosinska, X. Yin and D.B. Payne
}

\begin{abstract}
This paper reports on the architectural, protocol, physical layer and integrated testbed demonstrations carried out by the DISCUS FP7 consortium in the area of accessmetro network convergence. Our architecture modelling results show the vast potential for cost and power savings that node consolidation can bring. The architecture however also recognises the limitations of long-reach transmission for low latency 5G services, and proposes how to address such shortcomings in future projects. The testbed results, which have been conducted end-to-end, across access-metro and core, and have targeted all the layers of the network, from the application down to the physical layer, show the practical feasibility of the concepts proposed in the project.
\end{abstract}

Index Terms-access metro, network convergence, fixed mobile convergence, long-reach PON, flat optical core, optical island, 5G architecture, next generation multi wavelength PON.

\section{INTRODUCTION}

The DISCUS architecture was [1] developed within an EU FP7 Integrated Project with the rationale of growing network capacity for future services by 1000 times while solving the three main problems that such network growth will incur. These problems are: long term financial viability, energy consumption scalability and the digital divide between densely populated and sparse rural areas.

The solution developed within DISCUS is an architecture that minimises equipment and nodes in the network while maximally sharing the remaining infrastructure, equipment and nodes over as many customers as possible. It also proposed an integrated services solution that could deliver all foreseen services with either fixed access customer premises equipment (CPE) or wireless and mobile devices. The DISCUS architectural solution employs long reach passive optical networks (LR-PONs) connected to a small number of core nodes, which

M. Ruffini and D.B. Payne are with the CONNECT research centre headquartered at the University of Dublin, Trinity College, Ireland

M. Achouche is with III-V labs, France

A. Arbelaez and L. Quesada are with University College Cork, Ireland

R. Bonk and T. Pfeiffer are with Nokia-Bell-Labs Stuttgart, Germany

A. Di Giglio and M. Schiano are with Telecom Italia Tilab, Turin, Italy

N. J. Doran is with Aston University, Birmingham, U.K.

M. Furdek and L. Wosinska are with KTH, Stockholm, Sweden

R. Jensen and N. Parsons are with Polatis

J. Montalvo is with Telefonica I\&D, Madrid, Spain

C. Raack and R. Wessaly are with atesio, Berlin, Germany

H. Rohde is with Coriant, Munich, Germany

G. Talli and P. Townsend are with the Tyndall National Institute, University College Cork, Cork, Ireland

$\mathrm{X}$. Yin is with imec - Ghent University, IDLab, Ghent, Belgium

Manuscript received... we refer to as metro-core nodes or MC-nodes for short. These MC-nodes are interconnected by a set of optical light paths, where a light path is defined as a point to point optical connection between a pair of MC-nodes which has no packet processing at any intermediate nodes but only at the ingress and egress nodes. This set of light paths and the set of MCnodes, which we call an optical island, combined with the physical convergence of access and metro networks by use of the LR-PON, enables of the order 50:1 reduction in network traffic processing nodes in the network and produces a low cost and low energy consumption network architecture.

One of the main features of our approach is to consider the end-to-end view of the network, searching for solutions that are consistent across the access, metro and core networks, and could deliver a truly cost-effective design across the entire architecture.

This paper reports on the major conclusions from the project throughout its duration, and is organised as follows. The next section describes the overall optical access architecture and optimization studies that were carried out over a number of European countries. Section III provides details on the Metro-Core node architecture, which terminates the LR-PON connections on one side, while on the other side it interfaces with the flat optical core. Section IV provides some insight on reliability considerations of the DISCUS architecture, while Section V describes the operations and results of the testbed that demonstrates the end to end architecture and integrated multiple network layers, from the application down to the physical layer.

\section{OPTICAL ACCESS ARCHITECTURE}

In this section, we describe the optical access architecture that merges the access and the metro networks. The architecture enables the coexistence, management and control of different client systems on the same network infrastructure. Such a heterogeneous access network offers access for customers connected via both wireless and fixed network technologies and addresses the needs of residential, business and enterprise customers. Our approach is based on a high-split (physical split up to 512 with logical split up to 1024) and long-reach (up to $125 \mathrm{~km}$ ) passive optical network enabling a massive consolidation of central office sites to a small number of Metro-Core node locations. Our study shows that in principle about $98 \%$ of existing central office/local exchange sites can be bypassed and closed down with this architecture. We 
propose DWDM over the LR-PON with up to 50 upstream and 50 downstream wavelengths, respectively, in the C-band when using EDFA amplifiers in the LR-PON and many more wavelength channels when other optical bands are opened via the use of linear SOAs [2]. These wavelength channels can be dynamically assigned for different uses (depending on the business and ownership models) and customer demands in both downstream (DS) and upstream (US) directions. The target of this network scenario is the generation of a futureproof architecture that offers an end-to-end network scaling economically for a thousandtimesbandwidth increase compared to the customer usage and bandwidths used at the time the project started in 2012. Furthermore, the approach targets financial, environmental and social aspects providing minimum cost for delivered capacity, minimum power consumption and avoidance of a digital divide between customers located in urban and rural areas by providing much lower cost for fibre delivery into sparsely populated rural areas.

In Fig. 1 the general overview of the DISCUS end-to-end network solution is shown comprising a meshed flat optical core and long-reach PON which combines the access and metro networks. Electronic switching, routing and aggregation are performed in the Metro-Core nodes. The MC-node design is described in more detail in section III. MC-nodes are connected to the LR-PON access network via optical amplifier nodes (AN), which are typically located at what today are the central offices (CO) or local exchange (LE) sites. Selected $\mathrm{CO} / \mathrm{LE}$ sites can also be equipped with wireless network local processing functions if required for ultra-low latency services, as discussed later in the paper.

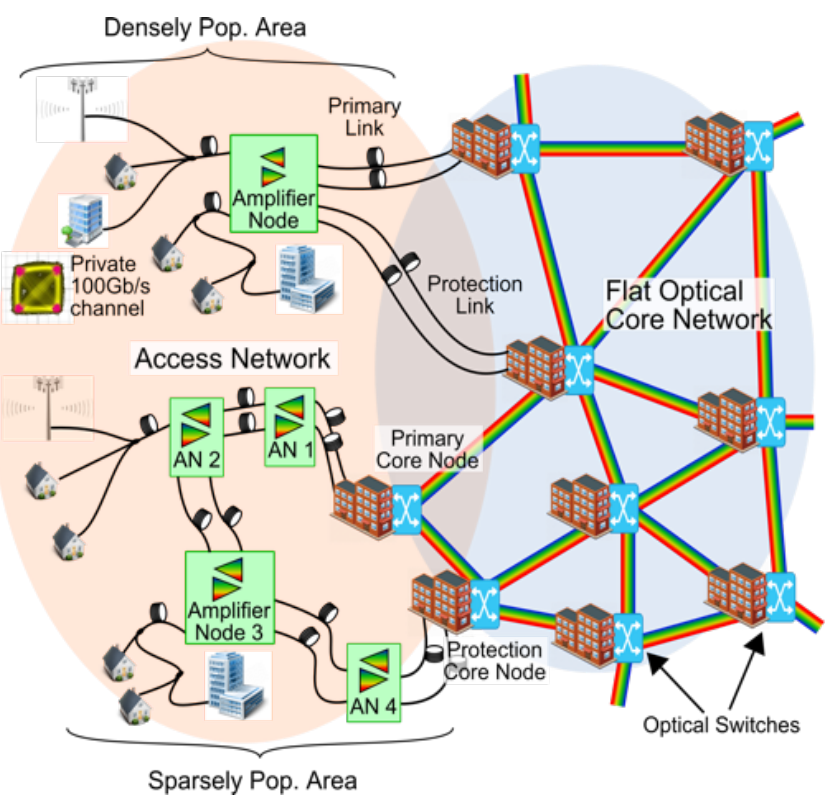

Fig. 1. Proposed LR-PON network concept with tree (top) and ring type (bottom) feeder topology, applicable for both urban and rural deployment scenarios

Each AN is dual-homed to a primary MC-node and to a second MC-node (named protection Core Node in the figure) for protection against feeder fibre and MC-node failure. It should be noticed that the second MC-node acts as protection node for some LR-PON links, but it is also a primary MC-node for others. LR-PON offers a general large scale split solution to support different network requirements in the optical distribution network (ODN), i.e. the section downstream of the ANs. For example, dense areas can have multiple splitters, based at the locations of the old CO/LE site, the cabinet site and the distribution point (DP) locations. More sparsely populated rural areas can add further splitting points to share fibres more efficiently in the longer length distribution network below the cabinet and DP positions. The split ratio on some branches can also be traded for increased reach in the ODN if required in very sparsely populated areas.

Although the logical PON layout is that of a tree topology, the real cable duct architecture (not shown in Fig. 1) used to connect the ANs to the pair of MC nodes is chosen to be predominantly a cable chain or open ring design which can re-use the configuration of earlier metro SDH network cabling structures. The use of cable chains minimises cable costs compared to tree networks where each LE has its own cabling network to its pair of MCs. The use of cable chains maximises the sharing of cable infrastructure and reduces fibre cost per customer site.

For sparsely populated rural areas we use amplifier chains, where the intermediate ANs provide additional amplification for the LR-PONs as the optical signals traverse the cable chain to the MC nodes [3]. This is shown in the bottom part of Fig. 1, where the chain of ANs (AN1 to AN4) is dual parented to two geographically separated nodes of the flat optical core network.

For the case of the UK mainland flat optical core network with $73 \mathrm{MC}$ nodes, the distribution of the shortest and longest distances along the cable chains of LEs to their dual parented MC-nodes (primary and secondary MC-node) is shown in Fig. 2 and Fig. 3 respectively. This is for a 73 MC-node flat optical core network. These results are for a particular set of metronetwork cable chains constrained by a maximum of 10 LEs per cable chain and a maximum LE to MC-node distance of $125 \mathrm{~km}$. We also utilize a high split ratio, i.e. 1:512, to reduce the number of PONs required to pass the customer base.

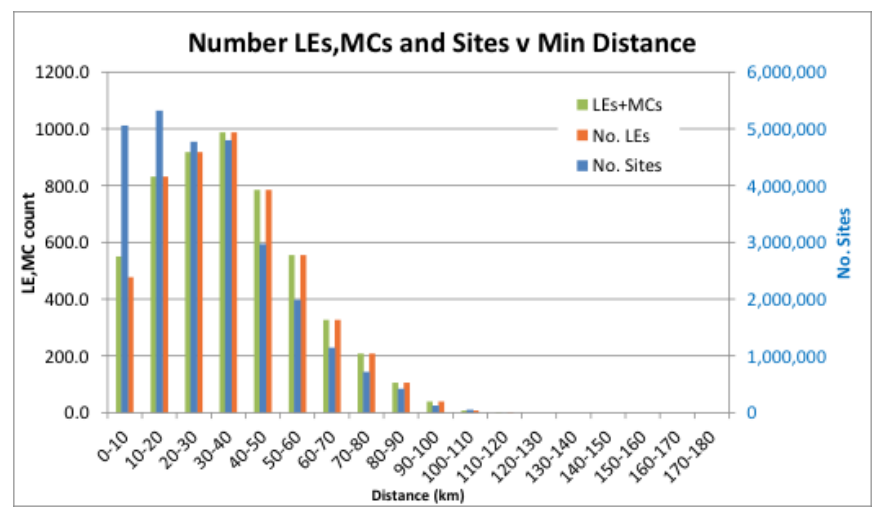

Fig. 2. The distribution of the shortest LE to MC node distances and the corresponding number of customer sites captured for the UK network

The distribution of the shortest distances (which will gener- 
ally be the distance of the LEs to the primary MC node) shows that the mean distance is $36 \mathrm{~km}$ with approximately $70 \%$ of customer sites within $40 \mathrm{~km}$ of the closest $\mathrm{MC}$ node. However the longest distance must be taken into account during systems design as, under fault conditions, ANs at the LE sites will switch to the longer secondary paths. In this case the mean distance is $76 \mathrm{~km}$ and only approximately $12 \%$ of customer sites would be within $40 \mathrm{~km}$ of the MC node. It should be stressed that the long reach of the LR-PON is required mainly in order to support the secondary protection paths.

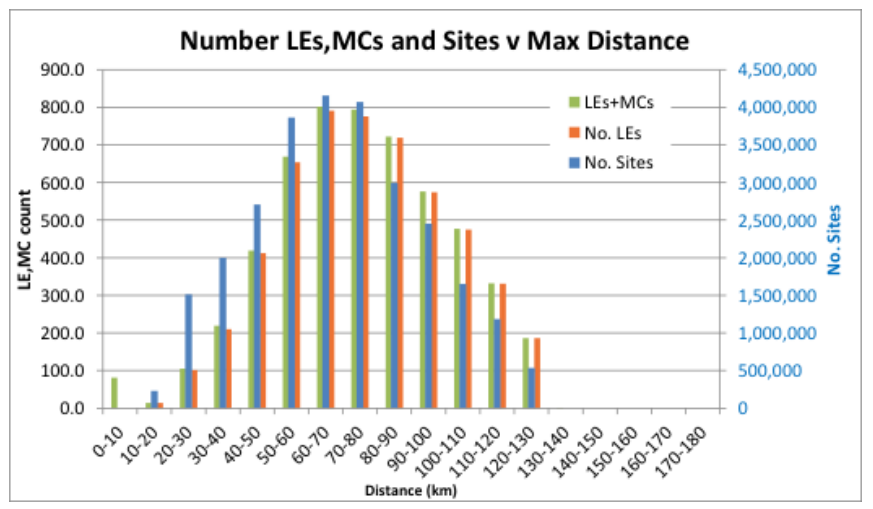

Fig. 3. The distribution of the longest LE to MC node distances and the corresponding number of customer sites captured for the UK network

When considering current requirements of front-hauling radio systems, the delay requirements imposed on the fixed backhaul network are quite stringent and limit fibre transmission reach to $40 \mathrm{~km}$. Although the shortest distribution indicates that the $40 \mathrm{~km}$ limitation can be met by a large proportion of the customer sites $(70 \%)$ when the protection path is taken into account only $12 \%$ of customer sites within $40 \mathrm{~km}$ of their more distant protection MC node can be supported. To facilitate the use of current front-hauling protocols, some electronic processing would therefore be needed at a large number of LE sites. Depending on the size and power consumption of this equipment it may not be possible to obtain the maximum extent of LE reduction that the DISCUS architecture could enable. This will increase both capital and operational expenditure compared to the minimum cost network.

How these additional costs are apportioned across the services requiring the front hauling technology is a question that was beyond the scope of the DISCUS project and is a question for future projects [4]. Similarly the important question of whether the stringent front-hauling requirements on the fixed network could be relaxed further to increase reach and enable the lower cost and lower power consumption fixed network as described by the DISCUS architecture should also be properly addressed in future projects on $5 \mathrm{G}$ and beyond.

The amplifier technology used to equip the ANs is either the erbium-doped fibre amplifier (EDFA) (shown in Fig. 4.a) for C-band operation or the semiconductor optical amplifier (SOA) (shown if Fig. 4.b) that can cover the entire wavelength band of the fibre in windows of about 60 to $100 \mathrm{~nm}$. In the case where the AN comprises the EDFA technology, one EDFA is used for DS and one for US direction. The reader can refer to
[2] for a detail description of the EDFA based AN. In the case where the AN is equipped with SOAs, an amplified splitter configuration is used [5]. For example, each branch of a 4:4 splitter is equipped with an SOA for DS and an SOA for US operation, i.e. in total 12 SOAs. Each of the SOAs offers a small-signal gain of about $15 \mathrm{~dB}$ and a noise figure of $7-8 \mathrm{~dB}$.

The network is designed to support a loss budget to bridge up to $125 \mathrm{~km}$ of single mode fibre, a split ratio of up to $1: 512$ (this can be greater for the chained AN node architecture), an optical signal-to-noise ratio (OSNR) larger than $15 \mathrm{~dB}$ in US direction including usual operational margins and penalties stemming from cross-talk or wavelength-drift under burstmode operation [6],[7] as well as from residual dispersion.

The access network architecture infrastructure supports different client systems and services on different wavelengths that can carry different bit rates and modulation formats. The residential customers are addressed with an on-off-keying (OOK) TDM/TDMA (time-division multiplexing)/(time-division multiple access) client system that symmetrically operates on DS and on US with 10Gbit/s. As discussed in the next-generation of passive optical network stage 2 (NG-PON2) [8], each of the optical network units (ONUs) contain wavelength tuneable elements in the transmitter and receiver sections. These elements enable a dynamic assignment of an independent DS/US wavelength pair to the customer premise equipment, i.e. assigning a customer to a particular service or network operator [9]. Additional wavelengths operating the same OOK TDMA protocol can be added to increase capacity as required for service and bandwidth growth. As a future upgrade path, a 40Gbit/s TDM-PON using electrical-duobinary (E-DB) modulation has been studied and results were presented in [10].

Large enterprise service needs that require very large dedicated bandwidths are offered by transportation of point to point core-bandwidth lightpaths over the LR-PON access network infrastructure reusing the PON fibre. Thus any dedicated bit rate from 10Gbit/s point-to-point (ptp) link using OOK modulation to a coherent solution at bit rates of 100Gbit/s using dual-polarization 28GBd quadrature phase shift keying (DPQPSK), in addition to shared PON protocol wavelengths, can be provided over the common LR-PON fibre infrastructure.

\section{A. Convergence of fixed and mobile networks}

The integration of the mobile services into the fixed network environment must support bandwidth and latency requirements for legacy wireless systems ( $3 \mathrm{G}$ and $4 \mathrm{G}$ ) as well as the requirements currently debated for future $5 \mathrm{G}$ deployments. Conceptually, we distinguish two wireless integration scenarios that differ in the radio access network (RAN) realization. The first approach is based on a centralized RAN architecture [11],[12] in which the digital baseband processing hardware, the baseband unit (BBU), is moved from the base stations to common central locations, serving a large group of remote radio heads (RRH) that then do not need much more hardware other than RF electronics. The optical transport between BBU and RRH is enabled by using the common public radio interface (CPRI). For the current CPRI front-haul approach, the analogue radio signals are digitized in the same format 
as they are transmitted over the air (except for down/upconversion). This requires a fixed line rate on the transport link, regardless of how much traffic information is actually conveyed.

In the following scenario, we target $1 \mathrm{Gbit} / \mathrm{s}$ IP peak traffic on the air interface per antenna sector at a RRH site. The resulting CPRI bit rates per sector are in the order of $15 \mathrm{Gbit} / \mathrm{s}$ : if we wish to use dedicated 10Gbit/s OOK wavelengths then CPRI compression must be used [13]. Note that in this scenario one transceiver (e.g. an ONU) per sector is employed for both macro cells (3 sectors / site that is 3 ONUs per RRH site) and small cells (1 sector / RRH site, one ONU per RRH). This particular front-haul approach is realized by a dense wavelength division multiplexing (DWDM) overlay in a similar way to that discussed in the NG-PON2 framework.

In the second approach, shown in Fig. 5, we propose to use the splitting of the wireless processing chain at a point, where the resulting interface capacity is dependent on the amount of traffic data to be transmitted over the air (user data or auxiliary signals like pilot tones). In this case the system can take advantage of user traffic dynamics, such that the optical link capacity benefits from statistical multiplexing effects. For obvious reasons such architectures are typically called midhaul with dual site processing or split processing [11]. With midhaul the optical transmission interface capacity dynamically ranges from zero (for no traffic) up to about $20 \%$ of the current CPRI rate in case of fully loaded radio channels. In this second scenario, we target an average IP bit rate of $1 \mathrm{Gbit} / \mathrm{s}$ per user at the air interface and an IP peak rate per sector of ¿10Gbit/s (maximum peak rate per user). In this case midhauling would require a maximum optical transmission rate of 30Gbit/s (down from $150 \mathrm{~Gb} / \mathrm{s}$ per sector that would be required for fronthaul). The use of TDM-PON seems very beneficial to establish a fronthaul link in such solutions (either 10Gbit/s OOK or 40Gbit/s E-DB).

One of the main drawbacks of front and midhaul links is their strict timing requirements in terms of total latency, timing accuracy and jitter accumulation, which need to be satisfied by the transport and networking mechanisms. Among the current radio access technologies, LTE imposes the most stringent requirements on transport latencies. This transmission latency relates to the $3 \mathrm{~ms}$ round trip time from the RRH to the BBU required by the hybrid Automatic repeat request (HARQ) function, which included travel time over the fibre as well as signal processing time in the optical and wireless systems. Depending on the optical transmission technology, the fibre length between BBU and RRH has to be limited to below 20 to $40 \mathrm{~km}$ (i.e., with a latency budget of a few hundred microseconds). Whereas with UMTS these constraints can be more relaxed, it is expected that for some applications in future $5 \mathrm{G}$ radio networks the latency constraints will be even tighter, e.g. in order to fulfil $1 \mathrm{~ms}$ latency requirements.

The long reach access network of the DISCUS architecture with dual parenting protection generally requires the RRH site to MC distance to be longer than $40 \mathrm{~km}$ as discussed above. Where longer distances are required, two approaches are possible. The first is to operate backhaul links using the legacy distributed RAN approach. The second, more oriented
TABLE I

NUMBER OF MC NODES NECESSARY TO CONNECT CUSTOMERS TO LR-PONS AND COMPARISON WITH CURRENT NUMBER OF LOCAL EXCHANGES

\begin{tabular}{|l||l||l||}
\hline Country & Number of current LE & $\begin{array}{l}\text { Number of DISCUS MC } \\
\text { nodes }\end{array}$ \\
\hline Ireland & 1204 & 12 \\
\hline UK & 5449 & 73 \\
\hline Spain & 8266 & 110 \\
\hline Italy & 10620 & 111 \\
\hline
\end{tabular}

towards 5G services approach, i.e., providing ultra-low latency and local communications, is to interconnect neighbouring remote sites on a local passive optical mesh network or to place small active nodes in the neighbourhood of remote sites (e.g. into the local exchange) offering local compute and/or higher layer processing capabilities (mini data centre in the LE). However this approach may mean fewer building closures and an increase in capital and operational expenditure which needs to be borne by those services requiring the low RTT of the $40 \mathrm{~km}$ distance limit and may mean they will no longer be financially viable. Again this is an area of further work for a future project.

\section{B. Architecture modeling}

In order to assess the benefit that the DISCUS architecture could achieve on a realistic network deployment we have carried out a number of major analytic and optimisation modelling activities that take into account data sources such as: population distribution, current location of local exchanges, location of residential and business premises and road layouts using open street maps for routing fibre connections.

One of the questions we have addressed is the potential node-consolidation ability of the LR-PON architecture in a number of different European countries. Turning local exchanges into remote amplifier nodes enables closing of the central office/local exchange buildings and only requires access to the cables and cable chamber with a small amount of electrical power for the optical amplifiers and minimal electronics for control, management and special functions at those sites. The switching and transmission equipment is consolidated into a relatively small number of metro-core nodes. The DISCUS architecture thus dramatically reduces the number of active nodes, and also bypasses the current metro transmission networks.

The optimization modelling behind the consolidation of MC nodes answers the following two central questions: firstly the minimum number of metro-core nodes needed to ensure dualhomed connectivity of all premises in a given country and their location; secondly the association between premises and LE sites and the set of metro-core nodes, together with the required fibre paths within the LR-PON reach.

While here we only report a few results of the modelling studies, the readers can refer to [14], [15], [16] for further details on the modelling and optimization tools used. Table I shows numbers for minimal MC node counts in the countries Italy, Spain, UK, and Ireland. These solutions provide disjoint redundant fibre paths between LE sites and two MC nodes 
and also balance the size of the MC nodes between 100 thousand and 1 million connected sites per node. The solution for the UK is shown in Fig. 6, depicting the MC node location and an example of the fibre links connecting RNs to MC nodes. It should be noticed that these models do not take into consideration potential limitations due to the latency constraints of some of the envisaged 5G services: this is left for future work.

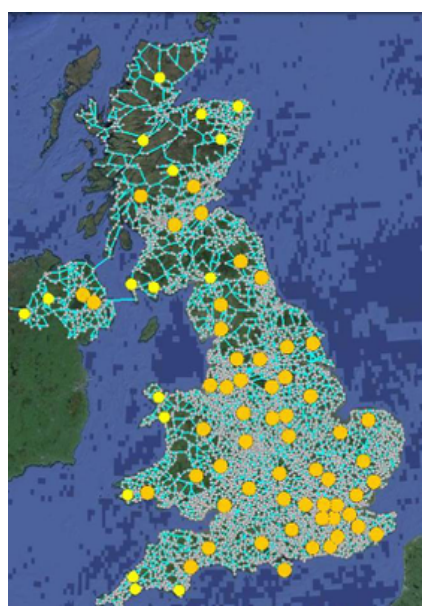

Fig. 6. MC node and fibre path allocation for a LR-PON deployment in the U.K.

\section{Metro-Core Design}

This sections describes more in detail the DISCUS metrocore node, whose overall architecture is presented in Fig. 7. The architecture is centred around a large port count optical fibre switch which constitutes the main, flexible interconnecting layer of the node, interconnecting access fibres, core fibres and any other electronic and optical equipment in completely reconfigurable arrangements.



Fig. 7. Metro-Core node architecture and control plane

The optical switch is implemented as a strictly nonblocking, hitlessly scalable 3 -stage optical switch fabric, capable of incrementally growing to over 24,000 ports with currently commercially available optical switching matrices [17]. After examining both dual-sided and single-sided Clos architectures, our analysis showed that the best choice is a single-sided folded 3-stage optical switch (thus in practice composed of two stages, as the first and third stages are merged) with an edge-fill strategy. This allows more flexibility with any-to-any port connectivity and simpler operations and management; it also allows some ports to only pass a single switch matrix if particularly low loss paths are required. The same single-sided reconfigurable switch is used in both the first and second stages. The single sided switch can also be partitioned so that not all ports are reachable from all other ports, as the overall reachability is not necessary in a practical implementation where, for example, only a small fraction of access fibres require direct connection to core fibres. Partitioning can be a convenient way to either grow the switch to larger sizes or reduce the switch matrix count for a switch of a given size.

In terms of performance, key optical switch design considerations for building multi-stage fabrics include: matrix size, total optical loss, reconfiguration speed, reliability and cost. DISCUS developed a very low loss single-sided 192port switching matrix using piezo beam steering elements, with median insertion loss and return loss values of $1.1 \mathrm{~dB}$ and $51 \mathrm{~dB}$ respectively. Using a 192 any-to-any switch building block, a single-sided 3-stage folded Clos can interconnect up to 12,288 bidirectional traffic lines or 6,144 bi-directional fibre pairs. In the first stage the 192 port reconfigurable switch used 64 poets for input traffic and 127 ports for connecting to second stage switches to insure strict non-blocking. The DISCUS partner Polatis has recently increased the switch matrix size to 384 ports, increasing a strictly non-blocking Clos single sided switch to 24640 ports.

A sample of the study results is shown in Fig. 8. This graph shows the number of switch edge ports that can be used for Metro-Core node traffic as a function of the total number of switch matrix ports used. It also shows a measure of portuse efficiency for the single-sided metro-core switch using both edge- and centre-fill scaling, to incrementally provide additional ports in a pay-as-you-grow fashion. Note that we define port use efficiency as the total number of switch matrix ports to the number of traffic ports on the edge of the switch. This asymptotically approaches 4.95 for a fully equipped switch and the higher the number the less efficient the switch port usage.

As expected, both the centre and edge-fill have very high port-use efficiency numbers when the switch is lightly loaded (inefficient port usage) and shows both approaches are very inefficient until the switch is $20-30 \%$ loaded. Ultimately the port-use efficiency number drops to 4.95 as expected for both edge and centre-fill when the switch is fully loaded. The edgefill method is clearly preferred over centre-fill because it uses fewer switch ports to support the same amount of traffic and has significantly better port-use efficiency at light to medium loading levels.

On the access side, the optical switch connects the WDM multiplexers facing the PON fibre to a number of electronic termination ports. It is envisaged that a number of these will 


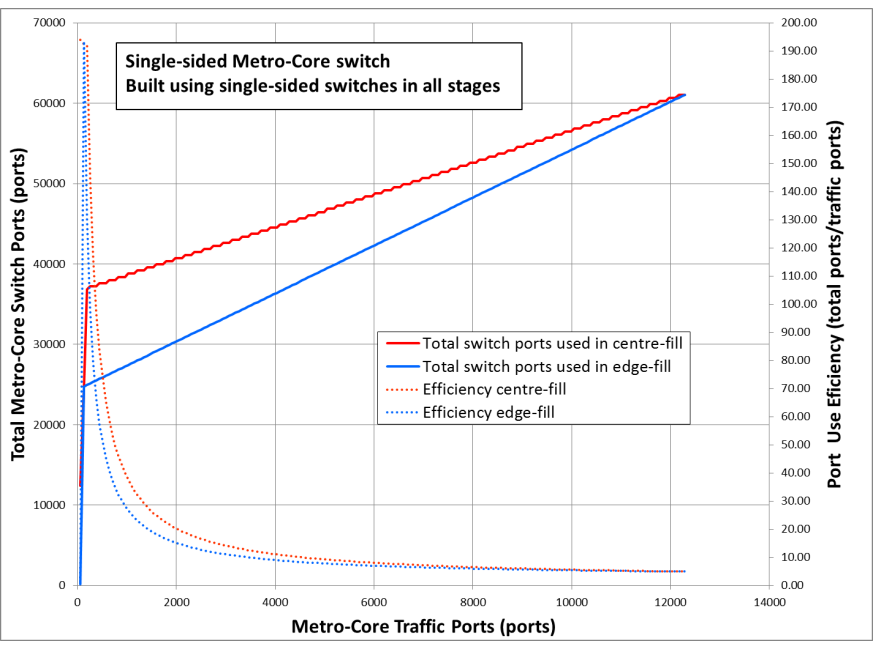

Fig. 8. Total ports used and port-use efficiency for single-sided Folded Metro-Core switch

be OLTs implementing protocols such as G-PON, XGS-PON, NG-PON2 and LR-PON protocol developed in the future. Some of the wavelength channels will be used however to provide dedicated virtual point-to-point links, at various rates (10G, 100G and beyond). It is also envisaged that some access connection could be switched directly to the core, passing through ancillary equipment that could provide functions from simple amplification to complete signal regeneration and protocol adaptation.

Access traffic is aggregated through an MPLS switch in the access side, which links to the IP/MPLS nodes from service providers. Where the node is also required to carry out core grooming, i.e., where the core part of the network is not implemented through a fully flat architecture, a core MPLS switch would carry out any core aggregation required.

As shown in Fig. 7 the SDN control plane system is developed through a hierarchical architecture, comprising:

- The access network controller, in charge of controlling the access network elements. On the south-bound interface the access controller acquires information and advises the optical line terminals (OLTs) to assign the necessary capacity. For example, user traffic flows can be prioritized based on the dynamic bandwidth assignment (DBA) protocol to establish an assured capacity per virtual local access network (VLAN) or to establish a dedicated wavelength for a particular customer demand based on a dynamic wavelength assignment (DWA) protocol.

- The core network controller, in charge of controlling the elements carrying out core transmission. The technologies considered in this architecture are Wavelength Switched Optical Network / Spectrum Switched Optical Network (WSON/SSON) networks which are based on the GMPLS distributed control plane. While there are valid examples of centralised controllers for core transport networks, such as the OpenSlice approach presented in [18], most transport networks are today based on the distributed GMPLS protocol. One of the main benefits is the lower protection time that a distributed control plane can currently provide. Although there is scope in the future for centralising core transport controllers, from an operator perspective it is important to demonstrate interoperability of state of the art SDN control planes with current GMPLS-based implementations. The core controller is in charge of receiving commands from the network orchestrator and transforming them in the southbound commands for the metro/core network elements.

- The network orchestrator, in charge of taking requests from the SP and translating them into high-level commands for the access and core network controllers. The end-to-end control plane architecture was demonstrated across a multi-laboratory, multi-project testbed case study, presented in [19], [20].

\section{Flat Optical Core Network}

The optical switching layer within MC-nodes allows interconnection to all other MC-nodes via transparent optical light paths that form a flat optical core network (Fig. 9). We call this transparently interconnected set of MC-nodes an optical island.

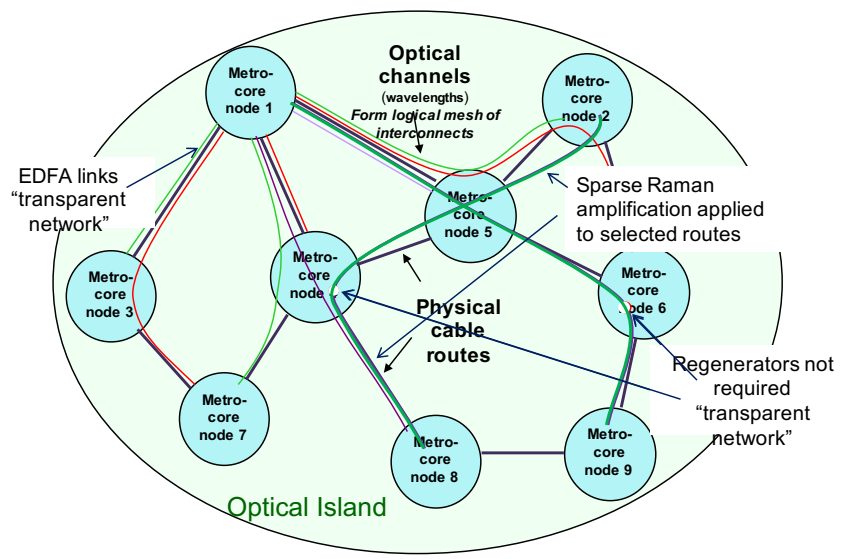

Fig. 9. Discus "Optical Island" which is a set of MC-nodes interconnected with transparent optical light paths. All nodes are at the same hierarchical layer

Fig. 10 shows that as user bandwidth increases a threshold is reached beyond which the flat optical core becomes lowest cost (and lowest power [21]), with respect to the hierarchical core and remains so for all subsequent bandwidth growth [23]. The case study considered in Fig. 10 was for a UK network containing $73 \mathrm{MC}$-nodes, where we considered an initial two-level hierarchical core with inner core network of 5 nodes (hence the label twolevel-5). The cost unit we use, ICU (IDEALIST Cost Unit), was defined in [22] by the FP7 IDEALIST project.

A strategy was also developed for evolving from the more conventional hierarchical core network used in today's network to the flat optical core network. The process is outlined in Fig. 11 which shows a simple 7 node core network with initially a two tier hierarchical core network with four outer core nodes and three inner core nodes (a) (the blue lines representing the inner core network).

As internode traffic increases some links between the outer core nodes have sufficient traffic to justify direct light path 


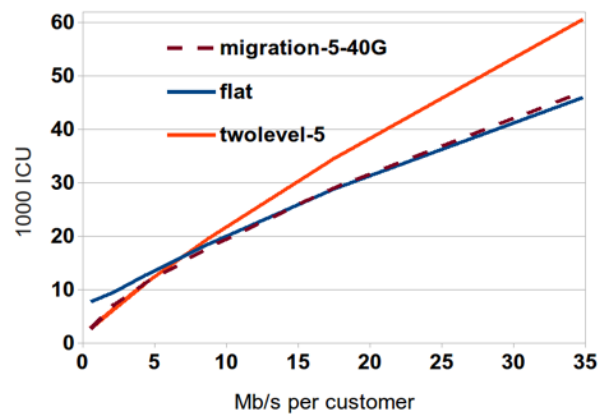

Fig. 10. Relative costs of Flat and Hierarchical core networks as a function of user bandwidth

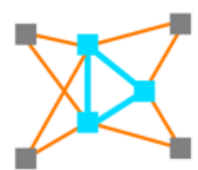

(a)

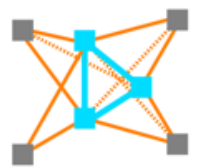

(b)

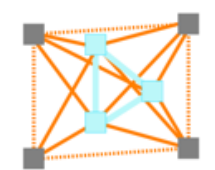

(c)

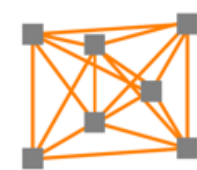

(d)
Fig. 11. Migration from two level hierarchy to flat optical core

interconnect (dotted orange lines) without going through the routers in the inner core nodes (b). These light paths are optically switched through transparent wavelength switching at the intermediate nodes. This is similar to providing optical express paths in today's networks on high traffic routes. This continues with more direct light paths established between nodes as traffic grows (c) until all nodes are interconnected with transparent light paths and the inner core nodes become part of the same hierarchical layer as the pouter core nodes forming a single layer optical island (d). The difference from today's network is that small nodes that would not form the MC-nodes of the optical island would be bypassed via the LRPON access network so that the total number of network nodes (both LE sites and small core nodes) are closed down leaving a small number of large MC-nodes fully interconnected with LR-PON networks that are dual parented onto those nodes.

\section{REliability CONSIDERATIONS}

This section describes network reliability studies carried out in the architecture, using simplified mathematical models to calculate reliability performance parameters (such as mean lifetime or mean time between failures (MTBF)) of each fibre section (feeder, distribution, drop) within the PONs. The source data for reliability figures was obtained by analysing data of subscribers complaints and the root causes of the service failures during the operation of a real FTTP deployment. The service interruptions caused by problems in the ODN are grouped into different categories depending on the fibre section where they originated. It must be taken into account that a failure in the feeder fibre affects all the subscribers in a PON ODN and a failure in a distribution fibre affects all the subscribers connected to its splitter branch. We use this model considering the number of reported problems (e.g., fibre cuts) in a FTTH deployment with 1:64 PONs formed by a cascade of 1:4 and 1:16 power splitters. Typical availabilities per $\mathrm{km}$ of fibre are much higher for feeder and distribution fibres (around 5 nines - 99.999\%), than for drop fibres (3 nines - 99.9\%), which are generally in a harsher physical environment and thus more prone to damage. However there are many more drop fibre than feeder sections in a PON (e.g., 64:1 in the case examined), thus although the probability of a failure (without considering ageing) in one feeder and in one distribution fibre (around 150 years and 250 years, respectively) is higher than in one single drop fibre (MTBF around 300 years) due to the longer length of a feeder and distribution section compared to the drop, we find that the majority of the failures (90\%) occur in the last drop fibres.

Compared to the (traditional) network architecture based on separate access and metro/aggregation segments (where the metro/aggregation segment provides its own resilience mechanisms), a LR-PON serving an equally large number of customers needs to offer similar reliability performance, so that business customers and backhauling of mobile services have similar levels of protection to the metro access networks of today. However, it can be shown that today's option based on unprotected GPON and separate metro/aggregation network has at least one order of magnitude higher connection availability compared to the unprotected LR-PON with unprotected feeder fibre between the MC-node and the AN. For this reason it is important to provide protection for the feeder fibre since it can be a single point of failure affecting a large number of users. In order to prevent such high impact failures, we have used dual homing to protect the feeder fibre in the long reach backhaul or feeder fibre segment of the LR-PON access network. This significantly improves the connection availability making it better that the one offered by unprotected GPON with a separate protected metro network. However as with the unprotected GPON case, it may not be sufficient for certain services and customers and additional options may be required.

In order to further increase the connection availability of LR-PON subscribers with very high reliability requirements, hybrid access ONUs connected by wireless links with the neighbouring ONUs can be deployed [24], [25], see Fig. 12.

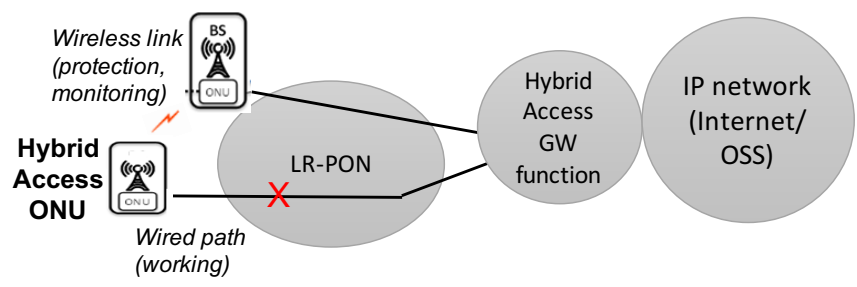

Fig. 12. Full protection in LR-PON for selected customers realized by using wireless link between related ONU

The wireless link offers a full protection to selected customers by providing a disjoint protection path in case of a drop and/or distribution fibre cut. It also allows reporting of ONU side optical time domain reflectometer (OTDR) measurements (if implemented) [26] to the operation and maintenance systems for detecting the location of fibre faults. This can reduce the mean time to repair (MTTR) and thus the overall access 
network availability is enhanced. It has been shown that such full protection can improve the connection availability in LRPON by several orders of magnitude, providing over 7 nines $(>99.99999 \%)$ availability in dense urban area and over 6 nines $(>99.9999 \%)$ in urban and rural areas. It should be noted that other communications paths between the related ONUs could be implemented such as additional fibre cables on separate physical paths.

\section{Vi. Multi-Service Testbed}

One of the aims of the DISCUS project was to demonstrate the architectural principles, the network solutions and the technology developed throughout the project in a live end-toend testbed. The demonstration testbed network was a multilayer system integrating all network layers, from the user application down to the LR-PON protocol and the optical transmission.

Fig. 13 illustrates the demonstrator network. It incorporates software defined networking (SDN) controlled Metro-Core nodes with a TDM-DWDM LR-PON connected to a primary core node, with a protection link to a secondary core node. More details on this experimental test-bed setup can be found in [2], [27]. By exploiting the dynamic allocation of DWDM channels, the LR-PON can support the convergence of a number of different user types and service demands, from residential users, which share a 10G PON channel, to business users, with options to rent dedicated 10G PON channels or high capacity $100 \mathrm{G}$ point-to-point (P2P) links.

The LR-PON demonstrator employed two different amplifier technologies in the AN: EDFAs, which are readily available and a well-known platform for amplification in Cband; and linear SOAs, which are a possible solution for integrated and compact ANs and extend operation outside the C-band [5].

The three different types of traffic, 10G PON, 100G P2P and front-haul, were demonstrated simultaneously over a dynamically reconfigurable TDM-DWDM PON physical layer with an ODN capable of supporting 512 users and a total LR-PON reach of up to $100 \mathrm{~km}$ [27]. The demonstrator also showed the capability of the system to support $40+40$ channels in up and downstream directions. The 10G PON channels used a $10 \mathrm{~Gb} / \mathrm{s}$ linear burst-mode receiver (LBMRx) [28] in the upstream link and implemented the PON protocol in the OLTs and ONUs using field programmable gate arrays (FPGAs). As an example Fig. 14 presents the bit error rate (BER) measurements obtained for the 10G PON channel in the upstream direction.

The BER is measured at the OLT in burst mode operation on $2 \mu s$ bursts generated by two different ONUs. The ODN loss was varied from 16 to $35 \mathrm{~dB}$ only for ONU 2 in order to vary the dynamic range (DR) of the burst powers from the two ONUs at the LBMRx, while the ONU 1 burst power is maintained constant at close to the LBMRx overload power (loud ONU) with an ODN loss of $16 \mathrm{~dB}$. Assuming a forward error correction (FEC) threshold of 1.1e-3, both designs in upstream direction are able to support an ODN loss of at least $28 \mathrm{~dB}$ with more than $3 \mathrm{~dB}$ system margin. An ODN loss of

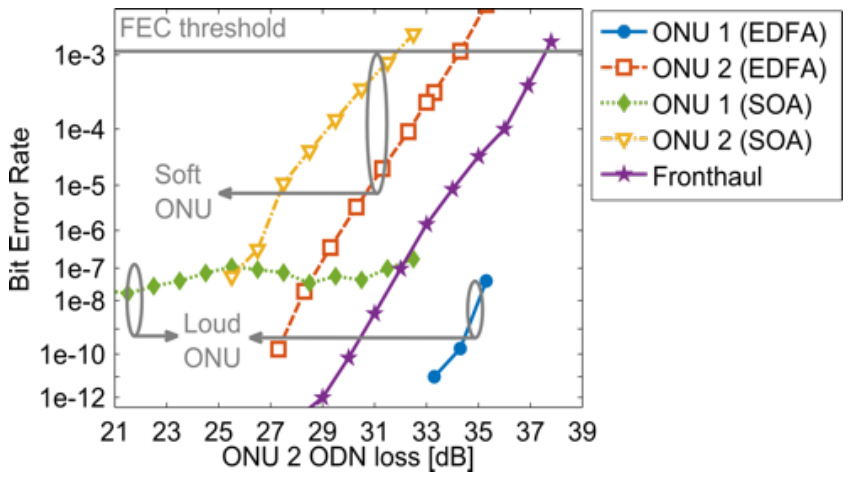

Fig. 14. BER of $10 \mathrm{~Gb} / \mathrm{s}$ PON downstream as a function of the ODN loss for the two amplifier options

$28 \mathrm{~dB}$ corresponds to a 128 split plus $12 \mathrm{~km}$ of fibre and to an overall split ratio of $512(128 \times 4$ due to the additional AN split), assuming end of life standard single mode fibre attenuation $(0.3 \mathrm{~dB} / \mathrm{km})$ and including realistic splitter excess losses in the ODN [29]. The BER is always below the FEC threshold for ONU 1 (below 1e-12 for most of the range in the EDFA case). The ONU 2 BER shows that the AN designs can support ODN losses of up to $31.5 \mathrm{~dB}$ and $34 \mathrm{~dB}$, corresponding to dynamic ranges of $15.5 \mathrm{~dB}$ and $18 \mathrm{~dB}$ respectively for the SOA and EDFA designs. Both designs can support a larger DR than that introduced by the non-uniform loss of the ODN splitters (up to $12 \mathrm{~dB}$ ), with the EDFA AN showing slightly better performance due to the lower noise figure (NF). A fronthaul wavelength channel terminated in the AN is also shown and can operate error free $\left(B E R<1 e^{-} 12\right)$ for an ODN loss of up to $28 \mathrm{~dB}$.

The SDN control plane can enable highly dynamic service and capacity provisions over the LR-PON in response to changing demand by implementing agents in the network elements. In the network test-bed demonstrated in [27] the SDN controller architecture, implemented in Ryu, follows the open network foundation (ONF) architecture using three main interfaces: the application-controller plane interface (ACPI) between the control plane and the application; the intermediate-controller plane interface (I-CPI) between the network orchestrator (NetO) and the access/core network controllers (NCs); and the device-controller plane interface (D-CPI) between the controllers and the physical devices [30]. The LR-PON protocol is a partial implementation of the XGPON standard, with the major differences being the longer distance and the higher split ratio supported.

Two different use cases have been demonstrated using the test bed in Fig. 13: an SDN-enabled fast protection mechanism and end-to-end service restoration in case of a primary link failure [31], [32]; and an SDN-enabled dynamic wavelength allocation (DWA) in response to an increased traffic demand [30]. In the fast protection use case the service restoration time is measured at the ONU when a failure event is caused by a cut in the backhaul link between the primary OLT and the AN [33]. Silence in the upstream activates a countdown timer in the primary OLT controller, which on expiry generates a failure detection and an in-band alarm to the Openflow access 


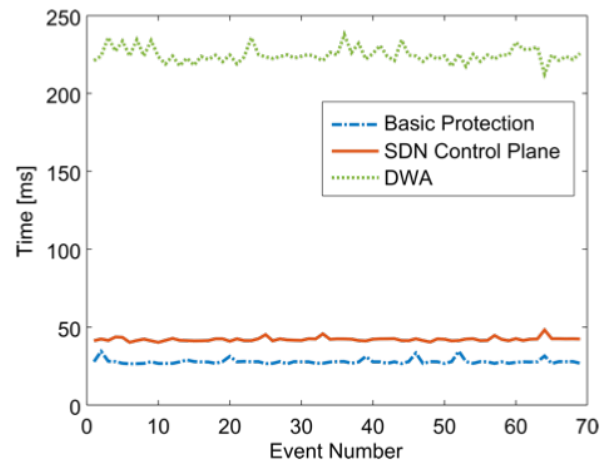

Fig. 15. Service restoration time for the protection mechanism and the DWA through the implemented SDN control plane

NC. The access NC alerts the Orchestrator, which provisions the protection path both on the access side, activating the dualhome link from the ONUs to a second MC node, and on the core side redirecting the data destined to $\mathrm{MC}$ node where the primary OLT resides towards the MC node where the backup OLT is located. An average service restoration time of $64 \mathrm{~ms}$ has been measured for the protection mechanism, where backup OLTs are shared among PONs in an N:1 scheme [27]. On the other hand, in the DWA use case the provisioning time is measured when, in response to an increase in traffic demand, the Orchestrator instructs the core and the access NCs to provision the new path, according to its knowledge of the full end-to-end network topology and the ONU traffic is moved to a different PON channel. This is accomplished by using a custom implemented physical layer operations, administration and maintenance (PLOAM) message used by the primary OLT to request the ONU to tune to a wavelength provisioned by the secondary OLT. We believe that the measured provisioning time of $225 \mathrm{~ms}$ could be reduced by an optimized design of communication interfaces between the ONU FPGA and the tuneable components [27]. The results of our experiments are reported in Fig. 15. Here the blue line represents the shortest protection time achieved by colocating orchestrator and controllers, thus removing any communication latency between the two. The red continuous line represents instead the results for the same protection experiment, where we have introduced more realistic communications latencies (4 $\mathrm{ms}$ ) between the OLT and the orchestrator and between the orchestrator and network controllers. Finally the top green line shows the results for the DWA experiment.

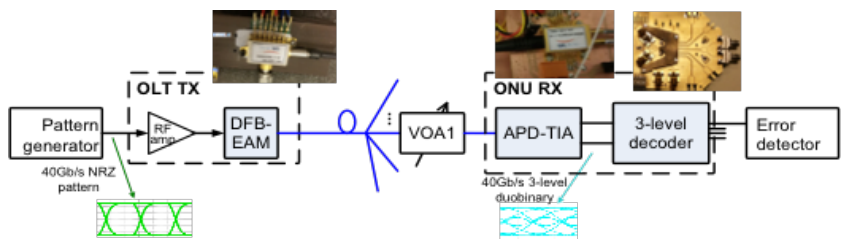

Fig. 16. A cost-effective implementation of a 40-Gb/s TDM-PON downstream link: utilizing an integrated high-power DFB-EAM TOSA in the OLT and an APD-based 3-level detection receiver in the ONU

A cost-effective 40-Gb/s single-carrier TDM-PON down- stream was also demonstrated utilizing an integrated highpower DFB-EAM (distributed feedback laser-electro absorption modulator) in the OLT and a 3-level detection receiver with an avalanche photodiode (APD) in the optical network unit (ONU). More details on the demonstration setup can be found in [10]. The proposed low-cost $40 \mathrm{~Gb} / \mathrm{s}$ downstream scheme is illustrated in Fig. 16. At the OLT, a compact and potentially low-cost integrated DFB-EAM transmitter optical sub-assembly (TOSA) is intensity modulated by a $40 \mathrm{~Gb} / \mathrm{s}$ $2^{7}-1$ pseudo random bit sequence (PRBS) NRZ signal. One important feature of such low-cost TOSA is the output optical power, which needs to be very high power to overcome the insertion loss of the EAM, while fulfilling the modulation speed and power budget requirement. In the ONU, the intensity modulated signal is low-pass filtered and converted into a 3level duo-binary signal. Unlike optical duo-binary modulation (ODB), the proposed scheme enables lower-bandwidth optical components (e.g., EML in the OLT and APD employed at the ONU), reducing the cost and power consumption.

A 3-level decoder IC was integrated in the ONU receiver, which also de-multiplexed the signal to 4 CML (chirpmanaged laser) output channels at $10 \mathrm{~Gb} / \mathrm{s}$. The BER measured at one of the $10 \mathrm{~Gb} /$ output of the 3-level decoder is shown in Fig. 17(a) for 3 different APD gain settings (i.e. different VAPD voltages). The optimal VAPD voltage was found to be $21.5 \mathrm{~V}$, where the APD gain was around $6.1 \mathrm{~dB}$. Further increasing the VAPD voltage above the optimum value reduces the receiver bandwidth, introducing more ISI in the output eyediagram and causing sensitivity deterioration. The measured sensitivity of $-19.6 \mathrm{dBm}$ at $\mathrm{BER}=1 \times 10^{3}$ resulted in a power budget of $23.4 \mathrm{~dB}$ in $\mathrm{B} 2 \mathrm{~B}$. The $40 \mathrm{~Gb} / \mathrm{s}$ 3-level duo-binary link was also evaluated as a function of dispersion. The measured power penalties versus various dispersion values are shown in Fig. 17(b) together with simulated results in [34]. The resulting power penalty in the range from $-215 \mathrm{ps} / \mathrm{nm}$ to $+128 \mathrm{ps} / \mathrm{nm}$ was $2.9 \mathrm{~dB}$, which is able to support more than $20 \mathrm{~km}$ of $\mathrm{SSMF}$ if dispersion pre-compensation is used.

\section{CONCLUSION}

This paper has demonstrated the concept of using longreach passive optical network for convergence of the access and metro networks into one integrated system, which enables massive consolidation of central offices. The work, carried out by the European DISCUS consortium, has targeted a complete analysis of this solution, from the physical layer design of system and novel components, to the protocol and control plane architecture design, to exploration of deployment optimisation over real country geographies and investigation of reliability options. The architecture modelling results have shown the vast potential for cost and power savings, associated with the high level node consolidation and reduction of electronic network cards enabled by the DISCUS architecture. The testbed results, which have been conducted end-to-end, across access-metro and core, and have targeted all the layers of the network, from the application down to the physical layer, have shown the practical feasibility of the concept. 


\section{ACKNOWLEDGMENT}

Financial support from EU FP7 project DISCUS (grant CNECT- ICT- 318137) and Science Foundation Ireland (SFI) (grant 13/RC/2077) is gratefully acknowledged.

\section{REFERENCES}

[1] M. Ruffini, et al., DISCUS: End-to-end network design for ubiquitous high speed broadband services. Proc. IEEE ICTON 2013.

[2] G. Talli, et al.,SDN Enabled Dynamically Reconfigurable High Capacity Optical Access Architecture for Converged Services. IEEE/OSA Journal of Lightwave Technology, Vol 35, No, 3, Feb. 2017.

[3] D. Carey et al., Dynamically Reconfigurable TDM-DWDM PON Ring Architecture for Efficient Rural Deployment, Proc. IEEE/OSA ECOC 2016, paper W.3.E.4.

[4] M. Ruffini, Multi-Dimensional Convergence in Future 5G Networks. IEEE/OSAJournal of Lightwave Technology, Vol 35, No, 3, Feb. 2017.

[5] R. Bonk et al., Wavelength-transparent long-reach-high-split TWsDMPON utilized by a non-gated parallel cascade of linear SOAs, Proc. IEEE/OSA ECOC 2014, paper P7.4.

[6] R. Bonk et al., The Underestimated Challenges of Burst Mode WDM Transmission in TWDM-PON, Elsevier Optical Fiber Technology Journal, Vol 26, part A, Dec. 2015.

[7] R. Bonk et al., Physical Media Dependent Challenges in US Path of Space and Service Converged TWDM-PON, Proc. IEEE/OSA OFC 2016, paper W2A.61.

[8] Recommendation G.989.2, 40-Gigabit-capable passive optical networks 2 (NG-PON2): Physical media dependent (PMD) layer specification, ITUT, December 2014.

[9] Th. Pfeiffer, A physical layer perspective on access network sharing, Elsevier Optical Fiber Technology Journal, Vol. 26, Part A, Dec. 2015.

[10] X. Yin et al., 40-Gb/s TDM-PON Downstream with Low-Cost EML Transmitter and 3-Level Detection APD Receiver, Prof. IEEE/OSA OFC 2016, paper Tu3C.1.

[11] T. Pfeiffer, Next Generation Mobile Fronthaul and Midhaul Architectures [Invited], IEEE/OSA Journal of Optical Communications and Networking, Vol. 7, N. 11, Nov. 2015.

[12] T. Pfeiffer, Next Generation Mobile Fronthaul Architectures, Proc. IEEE/OSA OFC 2015, paper M2J.7.

[13] S.-H. Park, O. Simeone, O. Sahin, S. Shamai, Fronthaul compression for cloud radio access networks. IEEE Signal Processing Magazine, Vol. 36, No. 6, Nov. 2014.

[14] N. Ascheuer, et al., A nation-wide optimization study on the consolidation of local exchanges using long-reach passive optical networks. Proc. IEEE ICTON 2014.

[15] C. Raack, et al., Nationwide deployment of (long-reach) passive optical networks: Computing the location and number of active nodes, Proc. ITG Fachtagung 2015.

[16] D. Mehta, et al., A scalable optimisation approach to minimising IP protection capacity for long-reach PON. Proc. IEEE ONDM 2014

[17] DISCUS Deliverable D6.5: Final report on the specification of the metro/core node architecture. July 2015.

[18] L. Liu, et al., OpenSlice: an OpenFlow-based control plane for spectrum sliced elastic optical path networks, OSA Optics Express, Vol. 21, No. 4, Feb. 2013.

[19] R. Vilalta, et al., The Need for a Control Orchestration Protocol in Research Projects on Optical Networking. Proc. IEEE EuCNC 2015.

[20] J. M. Gran Josa, et al.,End-to-end Service Orchestration From Access to Backbone. Proc. IEEE ONDM 2016.

[21] DISCUS Deliverable D2.8: DISCUS end-to-end techno-economic model, October 2015.

[22] IDEALIST Deliverable D1.1: Elastic Optical Network Architecture: reference scenario, cost and planning, June 2013.

[23] C. Raack, et al., Hierarchical Versus Flat Optical Metro/Core Networks: A Systematic Cost and Migration Study. Proc. IEEE ONDM 2016.

[24] DISCUS Deliverable D4.13: Resiliency in heterogeneous long reach access networks. June 2015

[25] Y, Yang, et al., Hybrid Fibre and Microwave Protection for Mobile Backhauling. IEEE/OSA Journal of Optical Communications and Networking, vol. 6 , no. 10, Oct. 2014 .

[26] H. Schmuck et al., Embedded OTDR techniques for cost-efficient fibre monitoring in optical access networks, Proc. IEEE/OSA ECOC 2006.

[27] G. Talli et al., Demonstration of SDN Enabled Dynamically Reconfigurable High Capacity Optical Access for Converged Services. Proc. IEEE/OSA OFC 2016, postdeadline paper PDPTh5B.1.
[28] S. Porto et al., Demonstration of $10 \mathrm{~Gb} / \mathrm{s}$ burst-mode transmission using a linear burst-mode receiver and burst-mode electronic equalization, Proc. IEEE/OSA OFC 2014, paper M3I.5G.

[29] ITU-T G989.2, 40-Gigabit-capable passive optical network 2 (NGPON2): Physical media dependent PMD layer specification. Dec. 2014.

[30] M. Ruffini et al., Software Defined Networking for next generation converged metro-access networks. Elsevier Optical Fiber Technology Journal, Vol. 26, Part A, Dec. 2015

[31] A. Nag, et al.N?1 Protection Design for Minimizing OLTs in Resilient Dual-Homed Long-Reach Passive Optical Network, IEEE/OSA Journal of Optical Communications and Networking, vol. 8, No. 2, February2016.

[32] S. McGettrick, et al., Experimental End-to-End Demonstration of Shared $\mathrm{N}: 1$ Dual Homed Protection in Long Reach PON and SDN-Controlled Core. Proc. IEEE/OSA OFC 2015, Paper Tu2E.5.

[33] S. McGettrick, et al., Experimental End-to-End Demonstration of Shared N:M Dual-Homed Protection in SDN-Controlled Long-Reach PON and Pan-European Core. IEEE/OSA Journal of Lightwave technology, vol. 34, No. 18, Sept. 2016.

[34] X. Yin, et al., Performance evaluation of single carrier 40-Gbit/s downstream for long-reach passive optical networks. Proc. IEEE ONDM 2014. 


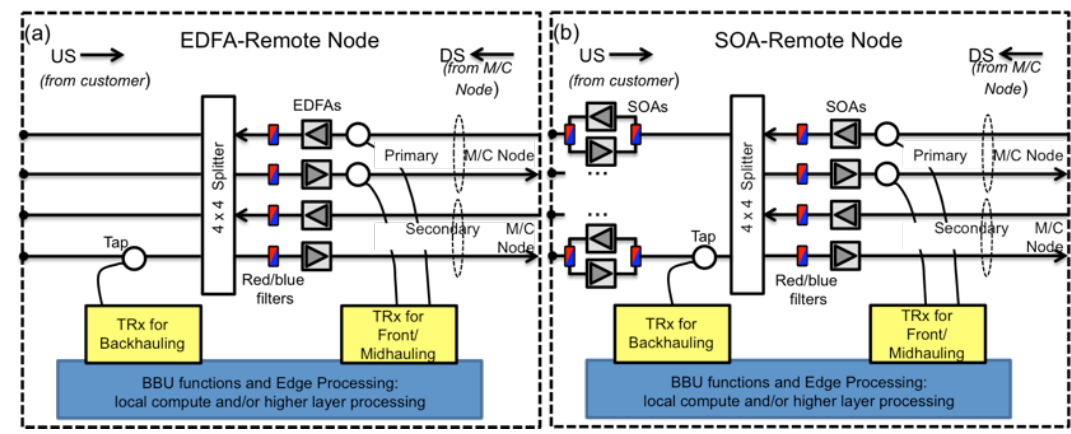

Fig. 4. Amplifier node configuration with (a) EDFA and (b) SOA amplification

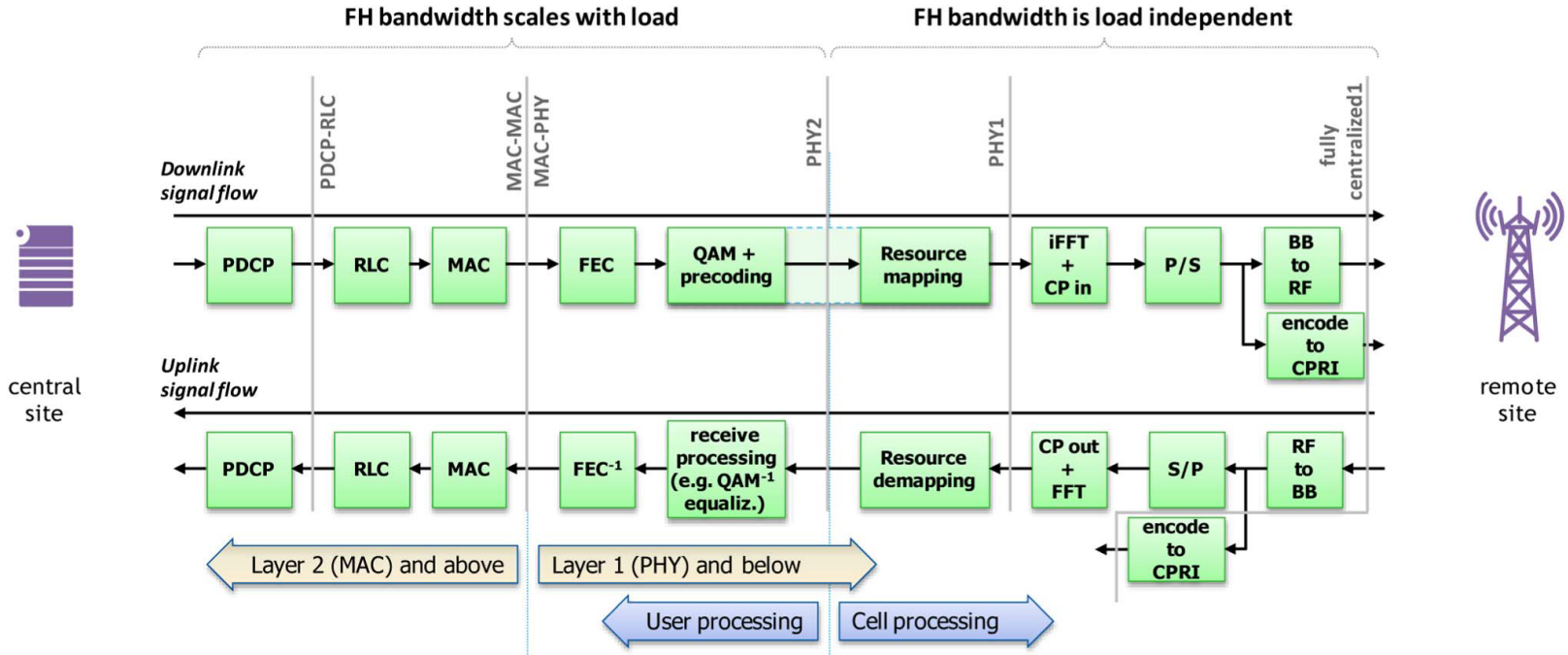

Taken from T. Pfeiffer, "Next Generation Mobile Fronthaul and MidhaulArchitectures [Invited]," J. Opt. Commun. Netw. 7, B38-B45 (2015).

Fig. 5. Layered LTE stack, showing potential splitting points for mid-haul architectures

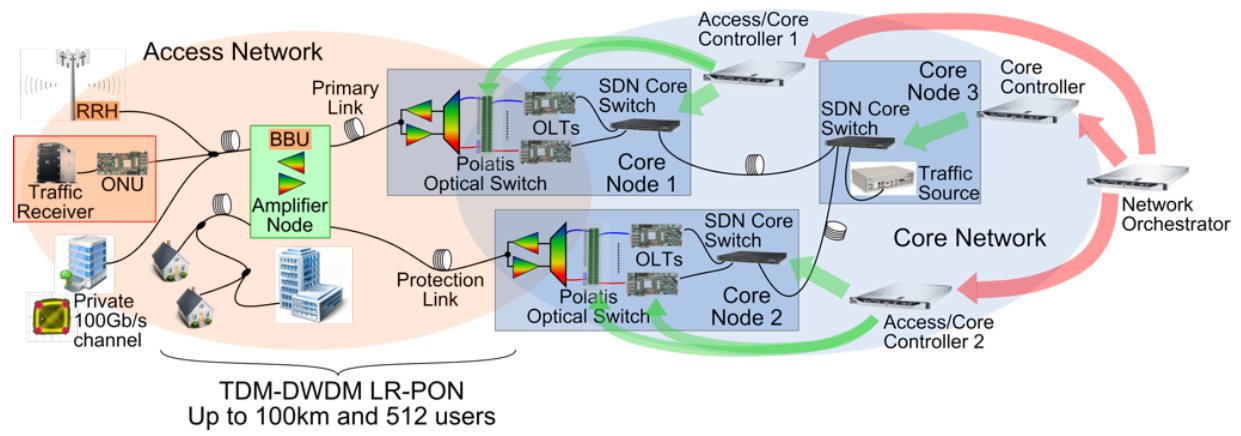

Fig. 13. Network level view of the DISCUS demonstrator 

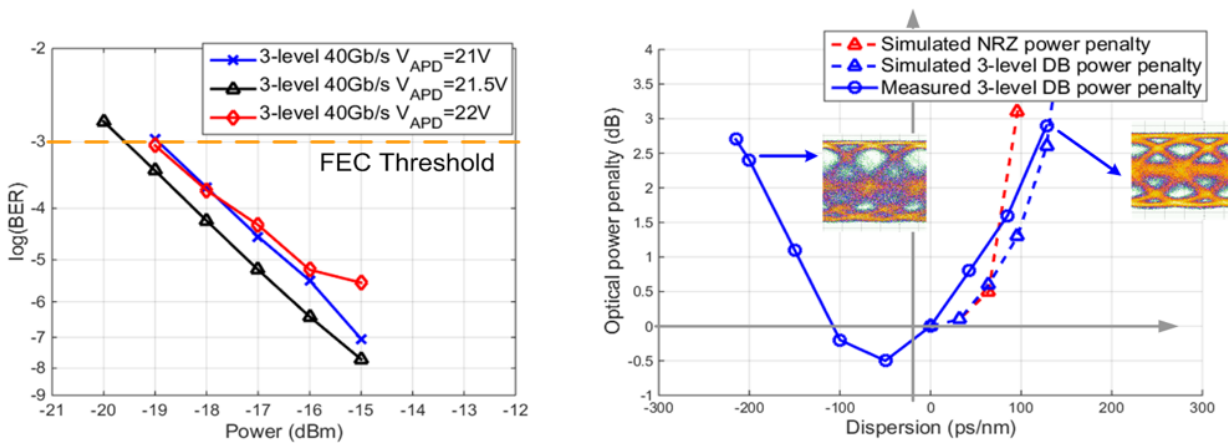

Fig. 17. Experimental results: (a) optimization of APD gain for $40 \mathrm{~Gb} / \mathrm{s}$ 3-level duo-binary downstream. (b) measured link power penalty in terms of dispersion in comparison of simulation results 